\title{
Effects of Cervical Spinal Cord Stimulation on Glucose Consumption in Patients with Posttraumatic Prolonged Unconsciousness
}

\author{
Narihito YaMAGUCHI, Hiroyasu SEKI*, Kiyonobu IKEDA, \\ and Junkoh YAMASHITA
}

\begin{abstract}
Department of Neurosurgery, Kanazawa University School of Medicine, Kanazawa; *Department of Radiology, Kanazawa Cardio-Vascular Hospital, Kanazawa
\end{abstract}

\begin{abstract}
The effects of cervical spinal cord stimulation (CSCS) on glucose consumption were examined in two patients with prolonged disturbance of consciousness due to head injuries. Several clinical parameters, including glucose consumption using positron emission tomography (PET) with [ $\left.{ }^{18} \mathrm{~F}\right] 2$-fluoro-2-deoxyD-glucose (FDG), were compared before and after CSCS. After a 4-month period of stimulation, one patient (Case 1) regained consciousness and began to speak, but the other patient (Case 2) showed no improvement in consciousness level. Computed tomography and magnetic resonance imaging showed Case 1 had no abnormalities in the thalamus and brainstem and no diffuse brain atrophy. Case 2 had a low density area in the left thalamus and enlargement of the aqueduct with diffuse atrophy of the left cerebral hemisphere. Cerebral blood flow studies and electrophysiological examinations revealed no remarkable change after CSCS. The PET study showed an increase in FDG uptake in the hypothalamus and thalamus in both patients, but an increase in FDG uptake in the left cingulate gyrus and left frontal lobe was observed only in Case 1. These observations suggest that activation of the ascending reticular activating system, hypothalamus, thalamus, cingulate gyгus, and frontal cortex, and the preservation of the fiber connections between the limbic system and the thalamus and hypothalamus are important for CSCS treatment to improve the level of consciousness.
\end{abstract}

Key words: cervical spinal cord stimulation, glucose consumption, positron emission tomography, prolonged unconscious state

\section{Introduction}

Advances in life-supporting medical care have lowered the mortality in patients with severe head injuries, but increased the number of patients with prolonged disturbance of consciousness. Some of these patients survive for months or years in a "persistent vegetative state" ${ }^{8)}$ that presents new medical and social problems. ${ }^{2.18}$ Medical treatment of these patients has rarely proved satisfactory. Surgical treatment for this state first used deep brain electric stimulation focused upon a non-specific thalamic nucleus. ${ }^{6,19}$ Deep brain stimulation has since been reported to be effective by several groups. Recently, cervical spinal cord stimulation (CSCS), usually used as a treatment for intractable pain, has been applied to prolonged unconsciousness patients with some success, ${ }^{4,11-13)}$ although the actual mechanism raising the consciousness level has not been clarified.

In this study, we compared clinical parameters, including results of positron emission tomography (PET) with $\left[{ }^{18} \mathrm{~F}\right]$ 2-fluoro-2-deoxy-D-glucose (FDG) before and after CSCS, to investigate the possible mechanism of action of CSCS in patients with prolonged unconsciousness.

Received September 9, 1994; Accepted February 20, 1995

Author's present address: N. Yamaguchi, M.D., Department of Neurosurgery, Asanogawa General Hospital, Kanazawa, Japan. 


\section{Materials and Methods}

Two patients who had been continuously unconscious for 4 months (Case 1) and for 6 years (Case 2) after head injuries were selected for treatment with CSCS using a neurostimulator (PISCES set) supplied by Medtronic Neuro (Minneapolis, Minn., U.S.A.). The distal end of the electrode was implanted in the epidural space at the C-2 level, and the reservoir was placed subcutaneously in the anterior chest wall. Stimulation $(10 \mathrm{~V}$, pulse width $0.2 \mathrm{msec}$, rate $45-100$ pulses $/ \mathrm{sec}$, amplitude $50 \%$ ) was started 7 days after implantation, and continued for 12 hours each day. Various clinical studies were performed before implantation and at least 14 days after the start of stimulation: computed tomography (CT), magnetic resonance (MR) imaging, electroencephalography (EEG), auditory brainstem response (ABR), somatosensory evoked potential (SEP), regional cerebral blood flow (rCBF) with ${ }^{99 m}$ Tc-hexamethyl propyleneamine oxime, and PET with FDG.

\section{Summary of Cases}

Case 1: A 27-year-old male became comatose with decorticate posture in response to noxious stimuli after head injury suffered in a traffic accident on November 4, 1991. CT on admission revealed acute brain swelling with small contusional hematomas in the bilateral frontal lobes and corpus callosum. Four months after the injury, he remained unconscious with tube feeding and total excretal incontinence. On March 13, 1992, the stimulation system was implanted. After 2 weeks of stimulation, he began to respond to orders for hand raising. After 4 months, tube feeding could be discontinued, and he began to speak a few broken words.

Case 2: A 16-year-old male suffered a head injury due to striking his head against a soccer goal post, and had been in a vegetative state for 6 years. On admission, he was in a deep coma with decerebrate posture due to acute epidural and subdural hematomas with traumatic contusion of the left hemisphere. Emergency surgery had removed the hematomas and contused tissues at admission, but his condition deteriorated, and he remained unconscious. On June 2, 1992, the CSCS system was implanted. After a 6-month period of stimulation, the spasticity of his extremities was slightly relieved, but no improvement in consciousness level was observed.

\section{Results}

Case 1: CT and MR imaging before CSCS disclosed subdural fluid collection in the bilateral frontal regions and contusional scars in the bilateral frontal lobes (Fig. 1). EEG revealed low voltage slow alpha waves with sporadic $4-6 \mathrm{~Hz}$ slow activities before CSCS, and increased fast alpha wave components after CSCS. The ABR pattern was normal before and after CSCS. An SEP study showed a normal pattern with right median nerve stimulation, but no components after $\mathrm{N}_{13}$ with left median nerve stimulation, and these findings were unchanged after CSCS. A rCBF study revealed hypoperfusion in the cerebellum, bilateral frontal lobes, and left temporal and occipital lobes. The mean $\mathrm{rCBF}$ value was 46 $\mathrm{ml} / 100 \mathrm{~g} / \mathrm{min}$ in the left hemisphere and $48 \mathrm{ml} / 100$ $\mathrm{g} / \mathrm{min}$ in the right hemisphere, with no significant difference before and after CSCS. The mean FDG uptake measured by PET was 89.3 and 88.3 counts per second (cps) $/ \mathrm{ml} / \mathrm{mCi} / \mathrm{kg}$ before and after CSCS, respectively. The FDG uptake increased in the brainstem, bilateral hypothalami and thalami, bilateral cingulate gyri, and left frontal cortex after CSCS. In contrast, the FDG uptake was reduced in the bilateral putamens and the bilateral temporal,

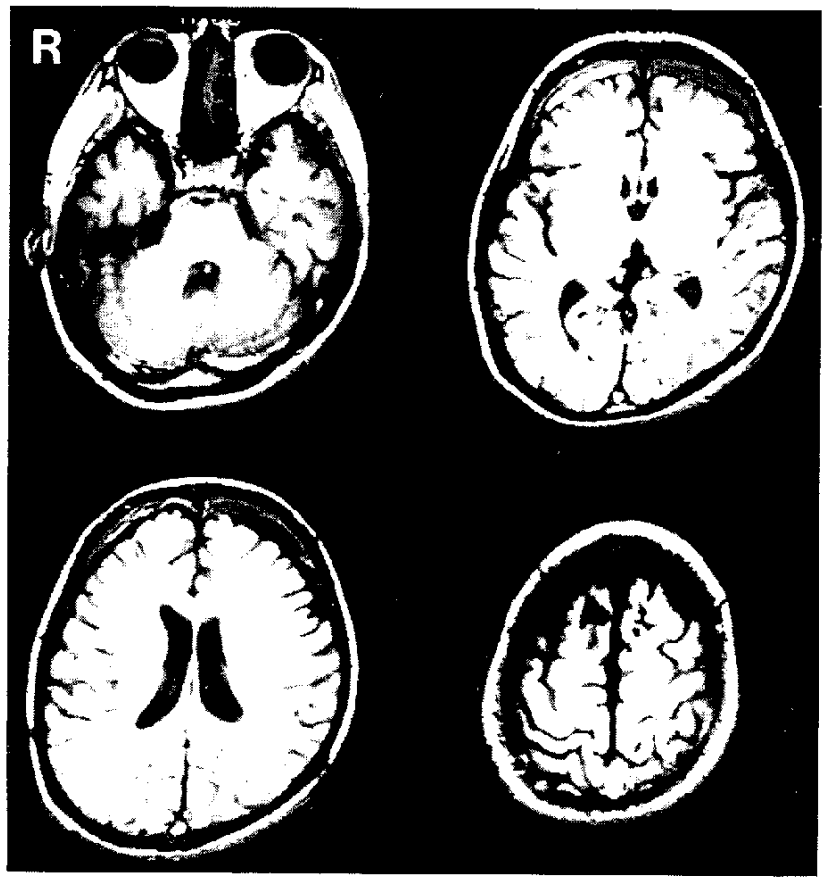

Fig. 1 Case 1. MR images 4 months after head injury showing fluid collection in the bilateral frontal regions, and contusional scars in the bilateral frontal lobes. 


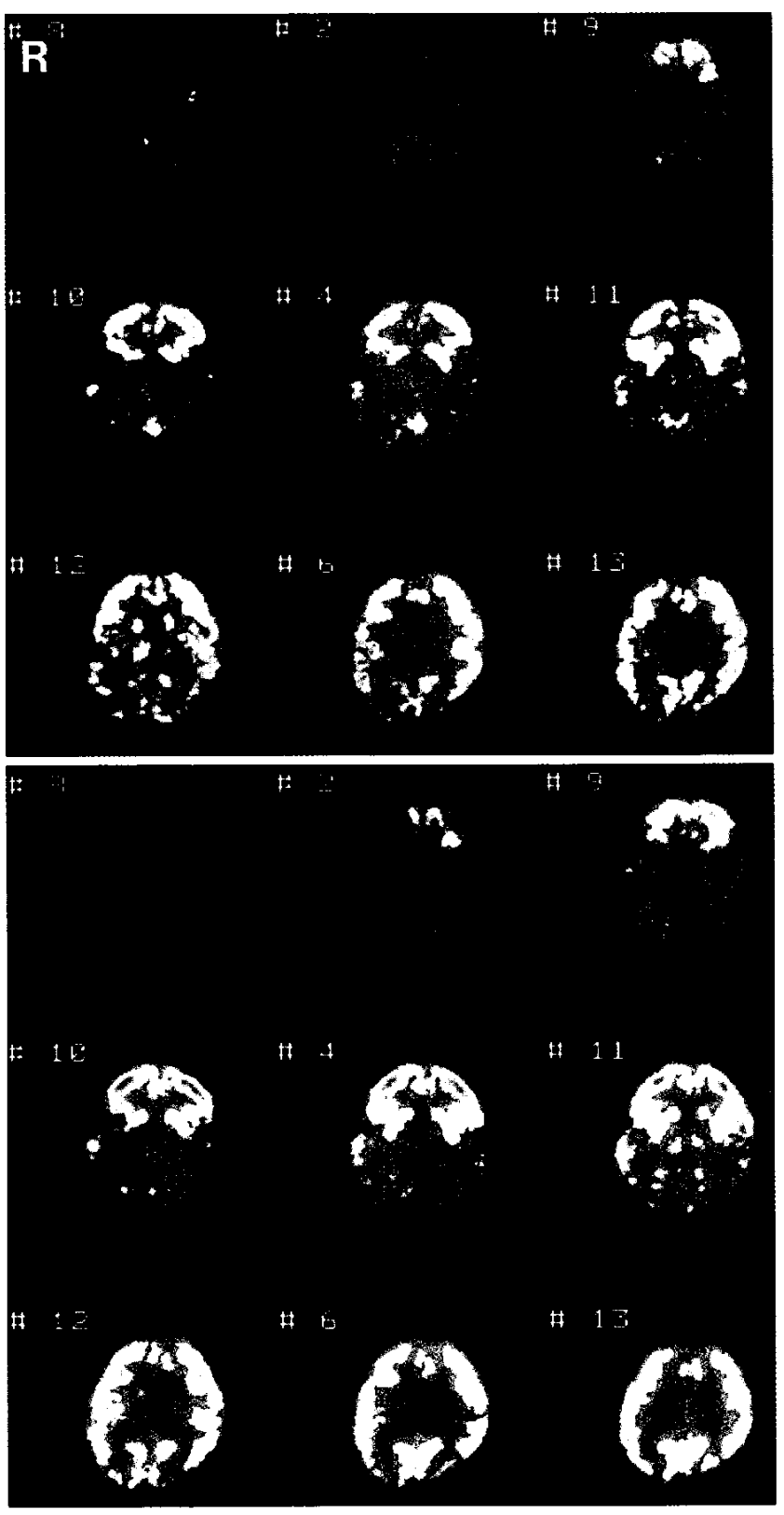

Fig. 2 Case 1. FDG PET scans before (upper panel) and after (lower panel) CSCS. Increased FDG uptake occurred in the bilateral hypothalami, thalami, and cingulate gyri, and left frontal cortex after CSCS.

parietal, and occipital cortices. The brainstem $(+19 \%)$, left hypothalamus $(+10 \%)$, left thalamus $(+16 \%)$, left cingulate gyrus $(+19 \%)$, and left frontal cortex $(+13 \%)$ demonstrated the largest increases in FDG uptake (Figs. 2 and 3).

Case 2: CT and MR imaging before CSCS revealed diffuse brain atrophy with contusional scars in the left hemisphere, a small low density area in the left thalamus, and enlargement of the aqueduct (Fig. 4). EEG before CSCS revealed slow waves with low activity and sporadic high voltage slow waves in the left hemisphere. The ABR study revealed a prolonged IIIrd wave and no components of the IVth or Vth wave. The SEP study revealed no components after $\mathrm{N}_{13}$ with bilateral median nerve stimulation. After CSCS, all these electrophysiological parameters showed no improvement. A rCBF study revealed remarkable hypoperfusion in the left hemisphere. The mean $\mathrm{rCBF}$ was $39 \mathrm{ml} / 100 \mathrm{~g} / \mathrm{min}$ in the left hemisphere and $52 \mathrm{ml} / 100 \mathrm{~g} / \mathrm{min}$ in the right hemisphere, with no significant difference before and after CSCS. The mean FDG uptake in the whole brain was 72.0 and $71.2 \mathrm{cps} / \mathrm{ml} / \mathrm{mCi} / \mathrm{kg}$ before and after CSCS, respectively. After CSCS, some regions of the brain showed an increase in FDG uptake, including the bilateral hypothalami (left, $+7 \%$; right, $+8 \%$ ) and thalami (left, $+7 \%$; right, $+3 \%$ ), but the bilateral cingulate gyri (left, $-7 \%$; right, $-4 \%$ ) and frontal cortices (left, $-11 \%$; right, $-5 \%$ ) showed a reduction of FDG uptake (Figs. 5 and 6).

\section{Discussion}

Although several successes using CSCS for the treatment of prolonged unconsciousness have been reported since 1987, the mechanism of CSCS on consciousness remains unclear, and the indications for this therapy have not been established. ${ }^{4-113)}$ We examined several physical and clinical parameters, including glucose consumption of brain with PET, to investigate the possible mechanism of CSCS on the arousal function.

CT and MR imaging showed no abnormalities in the thalamus and brainstem and no diffuse brain atrophy in the patient responding to CSCS (Case 1). The patient not responding to CSCS (Case 2) had a low density area in the left thalamus and enlargement of the aqueduct with diffuse atrophy of the left cerebral hemisphere. Diffuse bilateral cerebral atrophy, low density areas in the bilateral thalami, and diffuse brainstem abnormalities have all been suggested as possible factors in the failure of CSCS. ${ }^{4,12,13)}$

Some researchers have reported an improvement in EEG after CSCS, and we also observed an improvement in the EEG in Case 1. However, the EEG background activity is not predictive of the usefulness of CSCS, ${ }^{4)}$ and it is difficult to explain the mechanism of improvement of consciousness by only EEG findings or other electrophysiological examinations, including $\mathrm{ABR}$ and SEP. 


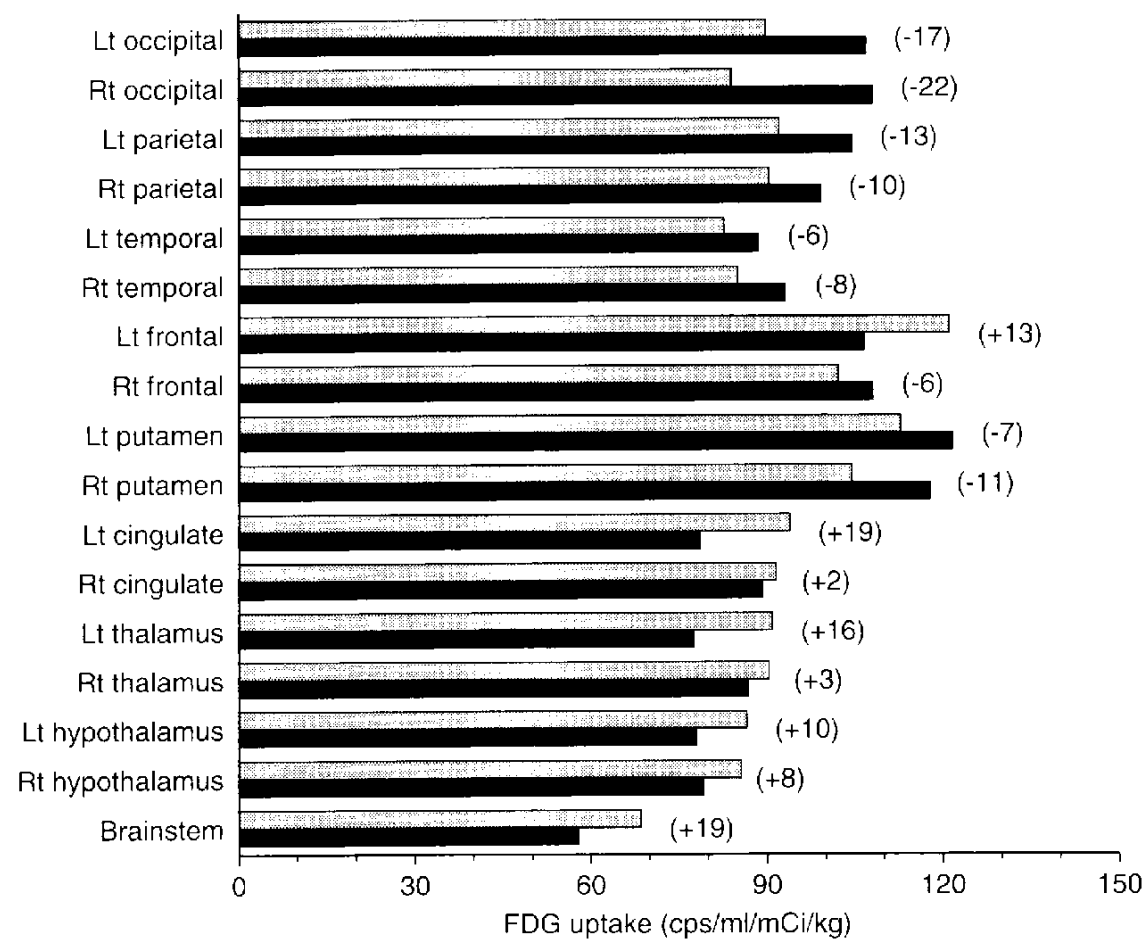

Fig. 3 Comparison of FDG uptakes before (closed column) and after (shaded column) CSCS in Case 1 . The brainstem, bilateral hypothalami and thalami, bilateral cingulate gyri, and left frontal cortex showed increases in FDG uptake. Numerals in parentheses represent \% changes from pre-CSCS value.

Hosobuchi $\mathrm{i}^{7}$ observed that CSCS produced a significant alteration in CBF values in patients, which persisted after cessation of CSCS, suggesting the involvement of humoral factors. Matsui and Hosobuchi ${ }^{14)}$ reported an increase in rCBF delayed by 1-2 hours after CSCS in animal models. Kanno et $a l{ }^{12)}$ directly examined catecholamines in cerebral spinal fluid after CSCS and reported that neurostimulation enhances the metabolism of catecholamines. These and our observations suggest that the change in $\mathrm{rCBF}$ after CSCS could result from the activation of glucose metabolism after neuropeptide secretion rather than being simply an axonal reflex.

The ascending reticular activating system is considered to lie within the brainstem, extending from the midbrain into the hypothalamus and thalamus. Structures within this region transmit diffuse physiological effects to the cerebral cortex that profoundly influence the arousal function. ${ }^{15)}$ At present, at least three principal ascending pathways from the reticular formation have been identified. One projects to the thalamic reticular nucleus and then to the cortex via a projection to the specific thalamic nuclei.
The second ascends through the hypothalamus to influence the basal forebrain structures, including the limbic system. The third is composed of axons of serotonin neurons of the midbrain raphe and norepinephrine neurons of the locus ceruleus, both of which provide diffuse, widespread innervation of the neocortex. ${ }^{9,17)}$

We observed no significant difference in the basic values of FDG uptake in the brainstem before CSCS between the two patients $(58.1$ and $57.4 \mathrm{cps} / \mathrm{ml} /$ $\mathrm{mCi} / \mathrm{kg}$ in Cases 1 and 2, respectively). An increase in FDG uptake in the brainstem after CSCS was observed in Case 1, but not in Case 2. The increase in FDG uptake occurred in the bilateral hypothalami and thalami, bilateral cingulate gyri, and the left frontal cortex in Case 1. In contrast, the FDG uptake was increased in the bilateral hypothalami and thalami, but reduced in the bilateral cingulate gyri and frontal cortices in Case 2 . From these results, we suggest that the fiber connections between the brainstem and the hypothalamus and thalamus, and between the hypothalamus and the frontal cortex via the limbic system, including the cingulate gyrus, were preserved in Case 1. We also suggest that the in- 


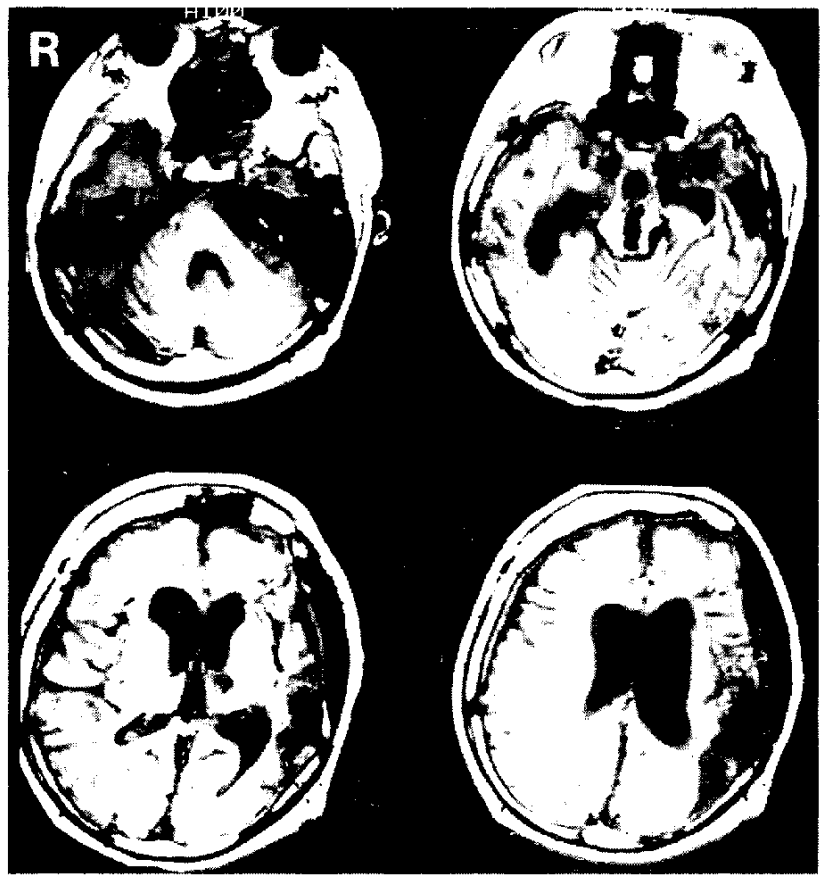

Fig. 4 Case 2. MR images 6 years after head injury showing diffuse brain atrophy with contusional scars in the left cerebral hemisphere, a low intensity region in the left thalamus, and enlargement of the aqueduct.

crease in FDG uptake in the hypothalamus and thalamus was caused by the indirect effects of CSCS, and the fiber connections between the hypothalamus and the limbic system were disrupted in Case 2.

Papez ${ }^{16)}$ proposed a closed circuit composed of the anterior nucleus, cingulate gyrus, hippocampus, and mamillary body, with impulses originating from visceral and somatic sensory fibers entering this closed circuit through the brainstem, hypothalamus, and mamillary body. ${ }^{10)}$ On the other hand, Cairns et $a l^{3)}$ suggested that impairment of either Vicq d'Azyr's bundle (mamillothalamic tract), which has connections with the cingulate gyrus, or fibers which originate in the hypothalamus and end in the medial nucleus of the thalamus are responsible for producing akinetic mutism.

Based on our results of glucose consumption with PET and previous anatomical studies, we suggest that the activation of both the ascending reticular activating system and the hypothalamus, thalamus, and cingulate gyrus, which has a closed connection with the frontal cortex and is an essential point for transmission of activity from the limbic system to the cerebral cortex, ${ }^{1,5\}}$ are required for CSCS treatment to be effective in the arousal of unconscious patients.
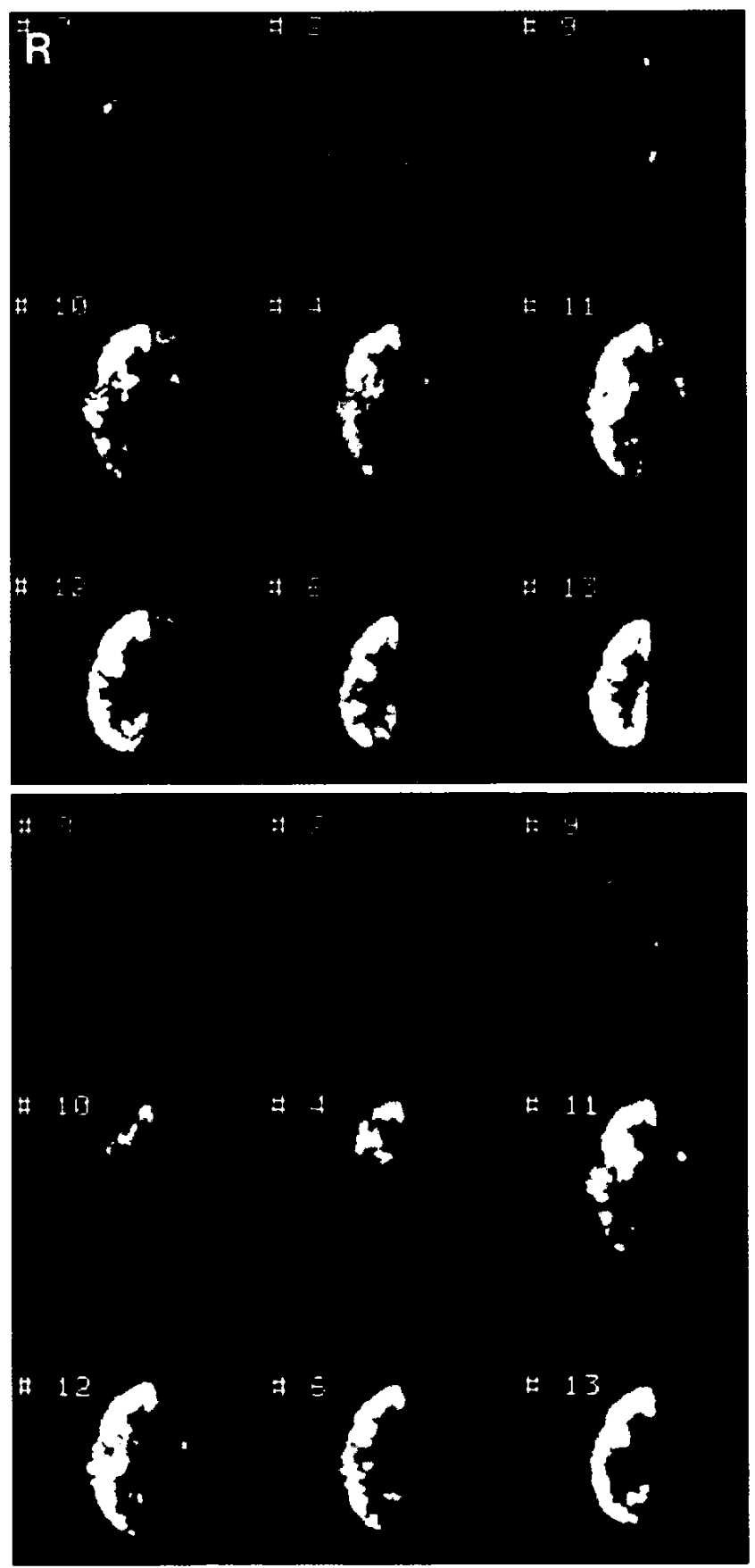

Fig. 5 Case 2. FDG PET scans before (upper panel) and after (lower panel) CSCS. Increased FDG uptake was observed in some regions of the brain, including the bilateral hypothalami and thalami, but FDG uptake was reduced in the bilateral cingulate gyri and frontal cortices.

At present, we suspect that the preservation of the fiber connection between the limbic system and the 


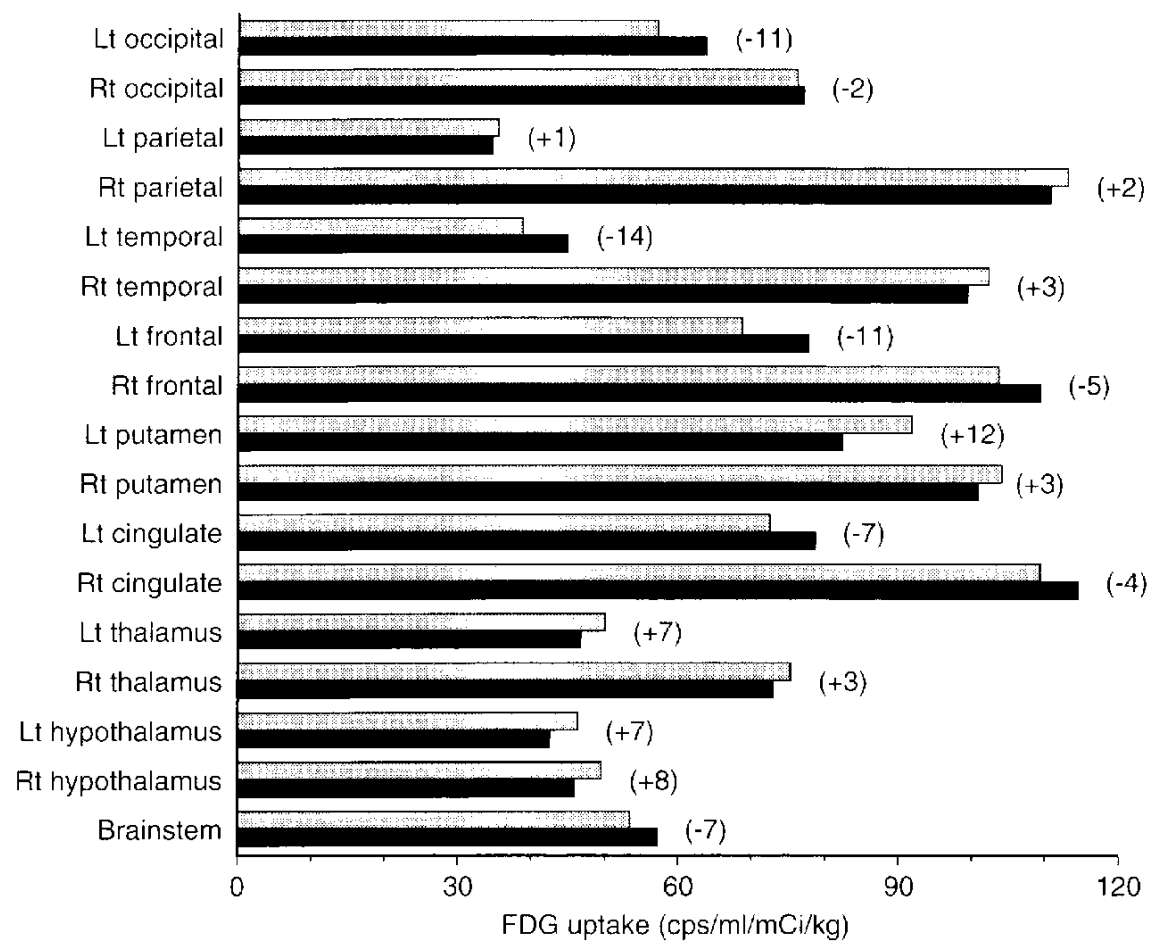

Fig. 6 Comparison of FDG uptake before (closed column) and after (shaded column) CSCS in Case 2. The bilateral hypothalami and thalami showed increased FDG uptake, but the bilateral cingulate gyri and the left frontal cortex showed reduced FDG uptake. Numerals in parentheses represent $\%$ changes from pre-CSCS value.

thalamus and hypothalamus is one of the most important factors.

\section{References}

1) Adey WR, Meyer M: An experimental study of hippocampal afferent pathways from prefrontal and cingulate areas in the monkey. $J$ Anat 86: 58-74, 1952

2) Bricold A, Turazzi S, Ferotti G: Prolonged posttraumatic unconsciousness. Therapeutic assets and liabilities. $J$ Neurosurg 52: 625-634, 1980

3) Cairns H, Oldfield RC, Pennybacker JB: Akinetic mutism with an epidermoid cyst of the 3 rd ventricle. Brain 64: 273-290, 1941

4) Funahashi $K$, Komai N, Ogura M, Kuwata $T$, Nakai $\mathrm{M}$, Tsuji N: Effects and indication of the spinal cord stimulation on the vegetative syndrome. No Shinkei Geka 17: 917-923, 1989 (in Japanese)

5) Glees P, Cole J, Whitty CWM: The effects of lesion in the cingular gyrus and adjacent areas in monkeys. J Neurol Neurosurg Psychiat 13: 178-190, 1950

6) Hassler R, Dalle Ore G, Dieckmann G, Bricolo A, Dolce G: Behavioural and EEG arousal induced by stimulation of unspecific projection systems in a patient with posttraumatic apallic syndrome. Electroenceph Clin Neurophysiol 27: 306-310, 1969
7) Hosobuchi Y: Electrical stimulation of the cervical spinal cord increases cerebral blood flow in humans. Appl Neurophysiol 48: 372-376, 1985

8) Jennett B, Plum F: The persistent vegetative state: A syndrome in search of a name. Lancet 1: 734-737, 1972

9) Jouvet M: Neurophysiology of the states of sleep. Physiol Rev 47: 117-119, 1967

10) Kaada BR: Cingulate, posterior orbital, anterior insular and temporal pole cortex, in Field J, Magoun HW, Hall VE (eds): Handbook of Physiology, vol 2, sect 1, Neurophysiology. Washington, DC, American Physiological Society, 1960, pp 1345-1350

11) Kanno $T$, Kamei $Y$, Yokoyama $T$, Jain VK: Neurostimulation for patients in vegetative status. PACE 10: 207-208, 1987

12) Kanno $T$, Kamei $Y$, Yokoyama $T$, Shoda $M$, Tsuji $H$, Nomura N: Effects of dorsal column spinal cord stimulation on reversibility of neuronal function. Experience of treatment for vegetative states, $P A C E 12$ : $733-742,1989$

13) Matsui $T$, Asano $T$, Takakura $K$, Yamada $R$, Hosobuchi Y: Beneficial effects of cervical spinal cord stimulation on patients with impaired consciousness: A preliminary report. PACE 12: 718-725, 1989

Neurol Med Chir (Tokyo) 35, November, 1995 
14) Matsui $T$, Hosobuchi $Y$ : The effects of cervical spinal cord stimulation (cSCS) on experimental stroke. $P A C E$ 12: 726-732, 1989

15) Moruzzi G, Magoun HW: Brain stem reticular formation and activation of the EEG. Electroenceph Clin Neurophysiol 1: 455-473, 1949

16) Papez JW: A proposed mechanism of emotion. Arch Neurol Psychiat 38: 725-740, 1937

17) Pulm F, Posner JB: The pathologic physiology of signs and symptoms of coma, in: The Diagnosis of Stupor and Coma, ed 3. Philadelphia, FA Davis, 1986, pp 1-30

18) Sazbon L, Groswasser Z: Outcome in 134 patients with prolonged posttraumatic unawareness. Part 1:
Parameters determining late recovery of consciousness. I Neurosurg 72: 75-80, 1990

19) Sturm V, Kuhner A, Schmitt HP, Assumus H, Stock $\mathrm{G}$ : Chronic electrical stimulation of the thalamic unspecific activating system in a patient with coma due to midbrain and upper brain stem infarction. Acta Neurochir (Wien) 47: 235-244, 1979

Address reprint requests to: N. Yamaguchi, M.D., Department of Neurosurgery, Asanogawa General Hospital, 83 Naka, Kosaka-machi, Kanazawa 920, Japan. 